\title{
Bäcklund Transformation and Exact Solutions to a Generalized $(3+1)$-Dimensional Nonlinear Evolution Equation
}

\author{
Yali Shen $(\mathbb{D}$ and Ying Yang \\ School of Mathematics and Information Technology, Yuncheng University, Yuncheng, Shanxi 044000, China \\ Correspondence should be addressed to Yali Shen; shenyali422@163.com
}

Received 2 March 2021; Revised 3 November 2021; Accepted 30 November 2021; Published 3 January 2022

Academic Editor: Zuonong Zhu

Copyright (c) 2022 Yali Shen and Ying Yang. This is an open access article distributed under the Creative Commons Attribution License, which permits unrestricted use, distribution, and reproduction in any medium, provided the original work is properly cited.

In this article, a generalized $(3+1)$-dimensional nonlinear evolution equation (NLEE), which can be obtained by a multivariate polynomial, is investigated. Based on the Hirota bilinear method, the $N$-soliton solution and bilinear Bäcklund transformation (BBT) with explicit formulas are successfully constructed. By using BBT, two traveling wave solutions and a mixed solution of the generalized $(3+1)$-dimensional NLEE are obtained. Furthermore, the lump and the interaction solutions for the equation are constructed. Finally, the dynamic properties of the lump and the interaction solutions are described graphically.

\section{Introduction}

With recent advancements in nonlinear science, the application of nonlinear evolution equations (NLEEs) has gained more prominence amongst mathematicians and physicists. The use of NLEEs is important because NLEEs can be used to describe many interesting nonlinear dynamic behaviors in nonlinear fields like optical fibers, plasma physics, atmospheric science, biologic nerve propagation, and marine science. Although the use of NLEEs is significant, the task of finding an explicit solution for the NLEEs has always been difficult and tedious. However, this task is also an important and meaningful exercise.

Many effective methods for solving NLEEs have been proposed by previous researchers [1-9]. For instance, Zhang et al. obtained the exact solutions for generalized nonlinear diffusion equations by using the separation of variables method [4]. He and $\mathrm{Wu}$ also used the Exp-function method to solve the nonlinear wave equations [5]. Xu et al. presented analytic solutions of the discrete PT-symmetric nonlocal nonlinear Schrödinger equation by Darboux transformation [9]. Amongst the proposed methods [1-9], the Hirota bilinear method is one of the most direct and effective methods for obtaining the exact solution of the NLEEs.
The most critical step in using the Hirota bilinear method is to transform the original nonlinear equation into a bilinear equation by introducing appropriate variable transformations. After obtaining the bilinear equation, the multisoliton solution of the original nonlinear equation can then be constructed. Interestingly, $\mathrm{Ma}$ et al. showed that multivariable polynomials can also produce Hirota bilinear equations [10]. The necessary criterion for the existence of a linear subspace for the exponential traveling wave solutions of the Hirota bilinear equation was given by Ma et al. [10]. Generally, the Hirota bilinear equations derived from multivariable polynomials have linear subspace solutions. [10]

By considering the multivariable polynomial given by

$$
P(x, y, z, t)=c_{1} x^{4}+c_{2} x t+c_{3} y^{2}+c_{4} y z+c_{5} z^{2},
$$

where $c_{i}(i=1, \ldots, 5)$ are arbitrary constants whose corresponding Hirota bilinear equation is

$$
\left(c_{1} D_{x}^{4}+c_{2} D_{x} D_{t}+c_{3} D_{y}^{2}+c_{4} D_{y} D_{z}+c_{5} D_{z}^{2}\right) f \cdot f=0,
$$

where $f=f(x, y, z, t)$ and $D$ is the Hirota bilinear operator [2]. 


$$
D_{x}^{m} D_{t}^{n} a \cdot b=\left.\left(\frac{\partial}{\partial x_{1}}-\frac{\partial}{\partial x_{2}}\right)^{m}\left(\frac{\partial}{\partial t_{1}}-\frac{\partial}{\partial t_{2}}\right)^{n} a\left(x_{1}, t_{1}\right) b\left(x_{2}, t_{2}\right)\right|_{\substack{x_{1}=x_{2} \\ t_{1}=t_{2}}}
$$

If the coefficients $c_{i}$ are chosen as $c_{1}=c_{2}=1$ and $c_{3}=c_{4}=c_{5}=0$, then (2) can be reduced to the bilinear form of the Korteweg-de Vries (KdV) equation. Furthermore, if the coefficients $c_{i}$ are set as $c_{1}=c_{2}=1, c_{3}=c_{5}=3$, and $c_{4}=0$, then (2) can be reduced to the bilinear form of $(3+1)$-dimensional Kadomtsev-Petviashvili

(KP) equation.

By introducing the variable transformation given by

$$
u=2(\ln f)_{x},
$$

(2) is then mapped to

$$
c_{1} u_{x x x x}+c_{2} u_{x t}+c_{3} u_{y y}+c_{4} u_{y z}+c_{5} u_{z z}+6 c_{1} u_{x} u_{x x}=0,
$$

where $f=f(x, y, z, t)$ and $u=u(x, y, z, t)$. Based on our literature review, (5) is a generalized $(3+1)$-dimensional NLEE which has not been presented by previous authors. Recently, many generalized NLEEs have attracted more attention as a result of their widespread application in many physical fields [11-14]. By investigating different kinds of generalized NLEEs, more general properties of the equations can be obtained.

This article is divided into sections and it focuses on the generalized $(3+1)$-dimensional NLEE represented by equation (5). In Section 2, the bilinear Bäcklund transformation (BBT) of equation (5) is constructed based on its Hirota bilinear equation. Armed with the BBT, the traveling wave solution and a mixed solution of (5) are obtained. In Section 3, the one-soliton, the two-soliton, and the threesoliton solutions are obtained, and then the $\mathrm{N}$-soliton solution is derived. In Section 4, the lump and the interaction solutions of (5) are given. The solutions also included some free parameters based on the bilinear form of (5). However, their dynamical behaviors are shown under some selected values of parameters. The article is then concluded with a summary and the implications of this research.

\section{Bilinear Bäcklund Transformation and Its Application}

Bäcklund transformation is important in the study of soliton systems. Some bilinear identities are given as follows [2]:

$$
\begin{aligned}
\left(D_{x}^{4} a \cdot a\right) b^{2}-\left(D_{x}^{4} b \cdot b\right) a^{2} & =2 D_{x}\left[\left(D_{x}^{3} a \cdot b\right) \cdot a b+3\left(D_{x}^{2} a \cdot b\right) \cdot\left(D_{x} b \cdot a\right)\right], \\
\left(D_{x} D_{t} a \cdot a\right) b^{2}-\left(D_{x} D_{t} b \cdot b\right) a^{2} & =2 D_{x}\left(D_{t} a \cdot b\right) \cdot b a, \\
\left(D_{x}^{2} a \cdot a\right) b^{2}-\left(D_{x}^{2} b \cdot b\right) a^{2} & =2 D_{x}\left(D_{x} a \cdot b\right) \cdot b a, \\
D_{t}\left(D_{x} a \cdot b\right) \cdot b a & =D_{x}\left(D_{t} a \cdot b\right) \cdot b a, \\
D_{x} a \cdot a & =0,
\end{aligned}
$$

where $a=a(x, t)$ and $b=b(x, t)$.

2.1. Bilinear Bäcklund Transformation. The BBT for (2) is then constructed and summarized as follows.

Theorem 1. If $f$ satisfies (2) as well as the following relations:

$$
\begin{aligned}
& B_{1} \hat{f} \cdot f=\left(c_{1} D_{x}^{3}+\lambda_{1} D_{y}+\lambda_{2} D_{z}+c_{2} D_{t}+\lambda_{3}\right) \hat{f} \cdot f=0 \\
& B_{2} \hat{f} \cdot f=\left(3 c_{1} D_{x}^{2}+\lambda_{4} D_{x}\right) \hat{f} \cdot f=0
\end{aligned}
$$

$$
B_{3} \hat{f} \cdot f=\left(c_{3} D_{y}+\lambda_{5} D_{z}+c_{4} D_{z}-\lambda_{1} D_{x}+\lambda_{6}\right) \hat{f} \cdot f=0,
$$

$$
B_{4} \widehat{f} \cdot f=\left(c_{5} D_{z}-\lambda_{2} D_{x}-\lambda_{5} D_{y}+\lambda_{7}\right) \hat{f} \cdot f=0,
$$

then $\widehat{f}$ also satisfies (2) with (11)-(14), where $\lambda_{i}(i=1, \ldots, 7)$ are arbitrary constants.

Proof. For the following function,

$$
P=\left(c_{1} D_{x}^{4}+c_{2} D_{x} D_{t}+c_{3} D_{y}^{2}+c_{4} D_{y} D_{z}+c_{5} D_{z}^{2}\right) \widehat{f} \cdot \widehat{f},
$$

we show that $\widehat{f}$ satisfying (11)-(14) can make $P=0$. 


$$
\begin{aligned}
& P f f=\left[\left(c_{1} D_{x}^{4}+c_{2} D_{x} D_{t}+c_{3} D_{y}^{2}+c_{4} D_{y} D_{z}+c_{5} D_{z}^{2}\right) \hat{f} \cdot \hat{f}\right] f f \\
& -\left[\left(c_{1} D_{x}^{4}+c_{2} D_{x} D_{t}+c_{3} D_{y}^{2}+c_{4} D_{y} D_{z}+c_{5} D_{z}^{2}\right) f \cdot f\right] \widehat{f} \hat{f} \\
& =c_{1}\left[\left(D_{x}^{4} \hat{f} \cdot \hat{f}\right) f^{2}-\left(D_{x}^{4} f \cdot f\right) \hat{f}^{2}\right]+c_{2}\left[\left(D_{x} D_{t} \hat{f} \cdot \widehat{f}\right) f^{2}-\left(D_{x} D_{t} f \cdot f\right) \hat{f}^{2}\right] \\
& +c_{3}\left[\left(D_{y}^{2} \widehat{f} \cdot \widehat{f}\right) f^{2}-\left(D_{y}^{2} f \cdot f\right) \hat{f}^{2}\right]+c_{4}\left[\left(D_{y} D_{z} \widehat{f} \cdot \widehat{f}\right) f^{2}-\left(D_{y} D_{z} f \cdot f\right) \widehat{f}^{2}\right] \\
& +c_{5}\left[\left(D_{z}^{2} \widehat{f} \cdot \widehat{f}\right) f^{2}-\left(D_{z}^{2} f \cdot f\right) \widehat{f}^{2}\right] \\
& \text { (6) }-(8) \\
& =2 c_{1} D_{x}\left[\left(D_{x}^{3} \hat{f} \cdot f\right) \cdot f \hat{f}+3\left(D_{x}^{2} \hat{f} \cdot f\right) \cdot\left(D_{x} f \cdot \hat{f}\right)\right]+2 c_{2} D_{x}\left(D_{t} \hat{f} \cdot f\right) \cdot f \hat{f} \\
& +2 c_{3} D_{y}\left(D_{y} \hat{f} \cdot f\right) \cdot f \hat{f}+2 c_{4} D_{y}\left(D_{z} \hat{f} \cdot f\right) \cdot f \hat{f}+2 c_{5} D_{z}\left(D_{z} \hat{f} \cdot f\right) \cdot f \hat{f} \\
& =2 D_{x}\left[\left(c_{1} D_{x}^{3} \hat{f} \cdot f+\lambda_{1} D_{y} \hat{f} \cdot f+\lambda_{2} D_{z} \hat{f} \cdot f+\lambda_{3} \hat{f} \cdot f\right) \cdot f \hat{f}+\left(3 c_{1} D_{x}^{2} \hat{f} \cdot f+\lambda_{4} D_{x} \hat{f} \cdot f\right) \cdot\left(D_{x} f \cdot \hat{f}\right)\right] \\
& +2 D_{x}\left(c_{2} D_{t} \hat{f} \cdot f\right) \cdot f \hat{f} \\
& +2 D_{y}\left(c_{3} D_{y} \hat{f} \cdot f+\lambda_{5} D_{z} \hat{f} \cdot f+\lambda_{6} \hat{f} \cdot f\right) \cdot f \hat{f}+2 D_{y}\left(c_{4} D_{z} \hat{f} \cdot f-\lambda_{1} D_{x} \hat{f} \cdot f\right) \cdot f \hat{f} \\
& +2 D_{z}\left(c_{5} D_{z} \hat{f} \cdot f-\lambda_{2} D_{x} \hat{f} \cdot f-\lambda_{5} D_{y} \hat{f} \cdot f+\lambda_{7} \hat{f} \cdot f\right) \cdot f \widehat{f} \\
& \text { (11) }-(14) \\
& =2 D_{x}\left(B_{1} \hat{f} \cdot f\right) \cdot f \widehat{f}+2 D_{x}\left(B_{2} \widehat{f} \cdot f\right) \cdot\left(D_{x} f \cdot \widehat{f}\right)+2 D_{y}\left(B_{3} \widehat{f} \cdot f\right) \cdot f \widehat{f} \\
& +2 D_{z}\left(B_{4} \hat{f} \cdot f\right) \cdot f \hat{f} \\
& \equiv 0 \text {. }
\end{aligned}
$$

Based on (9) and (10) respectively, it is clear that the coefficients of $\lambda_{i}(i=1,2,5)$ and $\lambda_{i}(i=3,4,6,7)$ are zeros. Hence, the proof of Theorem 1 is complete.

2.2. Application of BBT. The value $f=1$ is the solution of (2) and the corresponding original variable is $u=(\ln f)_{x}=0$. Due to $D_{x}^{n} a \cdot 1=\partial^{n} / \partial x^{n} a,(n \geq 1)$, BBT (11)-(14) associated with $f=1$ is reduced to the following equations:

$$
\left\{\begin{array}{l}
c_{1} \widehat{f}_{x x x}+\lambda_{1} \hat{f}_{y}+\lambda_{2} \widehat{f}_{z}+c_{2} \hat{f}_{t}+\lambda_{3} \widehat{f}=0 \\
3 c_{1} \hat{f}_{x x}+\lambda_{4} \widehat{f}_{x}=0 \\
c_{3} \hat{f}_{y}+\lambda_{5} \hat{f}_{z}+c_{4} \hat{f}_{z}-\lambda_{1} \hat{f}_{x}+\lambda_{6} \hat{f}=0 \\
c_{5} \hat{f}_{z}-\lambda_{2} \hat{f}_{x}-\lambda_{5} \hat{f}_{y}+\lambda_{7} \hat{f}=0
\end{array}\right.
$$

Different types of solutions to (5) can be obtained by substituting different forms of $\widehat{f}$ into (17).

2.2.1. Traveling Wave Solution. First consider the following form:

$$
\widehat{f}=1+\varepsilon e^{k x+l y+m z+\omega t},
$$

where $\varepsilon, k, l, m$, and $\omega$ are undetermined constants. By inserting (18) into (17) and assuming $\lambda_{3}=\lambda_{6}=\lambda_{7}=0$, these parameters can be determined by calculation as follows:

$$
\begin{aligned}
& \lambda_{2}=\frac{c_{3} c_{5} m+\lambda_{5}^{2} m+c_{4} \lambda_{5} m-\lambda_{1} \lambda_{5} k}{c_{3} k}, \quad \lambda_{4}=-3 c_{1} k, \quad l=\frac{\lambda_{1} k-\lambda_{5} m-c_{4} m}{c_{3}}, \\
& \omega=\frac{2 \lambda_{1} \lambda_{5} m k-c_{1} c_{3} k^{4}+c_{4} \lambda_{1} m k-\lambda_{1}^{2} k^{2}-c_{3} c_{5} m^{2}-\lambda_{5}^{2} m^{2}-c_{4} \lambda_{5} m^{2}}{c_{2} c_{3} k} .
\end{aligned}
$$


Therefore,

$$
u=\frac{2 k \varepsilon e^{k x+\left(\left(\lambda_{1} k-\lambda_{5} m-c_{4} m\right) / c_{3}\right) y+m z+\left(\left(2 \lambda_{1} \lambda_{5} m k-c_{1} c_{3} k^{4}+c_{4} \lambda_{1} m k-\lambda_{1}^{2} k^{2}-c_{3} c_{5} m^{2}-\lambda_{5}^{2} m^{2}-c_{4} \lambda_{5} m^{2}\right) / c_{2} c_{3} k\right) t}}{1+\varepsilon e^{k x+\left(\left(\lambda_{1} k-\lambda_{5} m-c_{4} m\right) / c_{3}\right) y+m z+\left(\left(2 \lambda_{1} \lambda_{5} m k-c_{1} c_{3} k^{4}+c_{4} \lambda_{1} m k-\lambda_{1}^{2} k^{2}-c_{3} c_{5} m^{2}-\lambda_{5}^{2} m^{2}-c_{4} \lambda_{5} m^{2}\right) / c_{2} c_{3} k\right) t}}
$$

This solves (5), where $\varepsilon, m, \lambda_{5}, \lambda_{7}$, and $c_{i}(i=1,4,5)$ are arbitrary constants and $k c_{2} c_{3} \neq 0$. Solution (20) is a onestripe soliton solution of (5).

By taking a simple polynomial solution,

$$
\widehat{f}=k x+l y+m z+\omega t,
$$

where $k, l, m$, and $\omega$ are constants to be determined. Similarly, by substituting (21) into (17) and supposing $\lambda_{i}=0(i=3,6,7)$, a direct computation gets

$$
\begin{aligned}
\lambda_{2} & =\frac{c_{3} c_{5} m+\lambda_{5}^{2} m+c_{4} \lambda_{5} m-\lambda_{5} \lambda_{1} k}{c_{3} k}, \\
l & =\frac{\lambda_{1} k-\lambda_{5} m-c_{4} m}{c_{3}}, \\
\lambda_{4} & =0, \\
\omega & =\frac{2 \lambda_{1} \lambda_{5} m k+c_{4} \lambda_{1} m k-\lambda_{1}^{2} k^{2}-c_{3} c_{5} m^{2}-\lambda_{5}^{2} m^{2}-c_{4} \lambda_{5} m^{2}}{c_{2} c_{3} k},
\end{aligned}
$$

with $k c_{2} c_{3} \neq 0$ and $m, \lambda_{1}$, and $\lambda_{5}$ are arbitrary constants.

Hence,

$$
u=\frac{2 k}{k x+l y+m z+\omega t}
$$

is a rational solution to (5), and parameters $k, l, m$, and $\omega$ satisfy (22).

2.2.2. Mixed Solution. $\widehat{f}$ is now taken as a combination of a polynomial function and cosine function:

$$
\begin{aligned}
\widehat{f}= & \left(k_{1} x+l_{1} y+m_{1} z+\omega_{1} t\right) \\
& +\varepsilon_{1} \cosh \left(k_{2} x+l_{2} y+m_{2} z+\omega_{2} t\right),
\end{aligned}
$$

where $\varepsilon_{1}, k_{i}, l_{i}, m_{i}$, and $\omega_{i}(i=1,2)$ are constants to be determined. By inserting (24) into (17), these parameters can be determined by calculation as follows:

$$
\begin{aligned}
& \lambda_{2}=\frac{2 c_{5} \lambda_{1}}{c_{4} \pm \sqrt{c_{4}^{2}-4 c_{3} c_{5}}}, \\
& \lambda_{5}=\frac{-2 c_{3} c_{5}}{c_{4} \pm \sqrt{c_{4}^{2}-4 c_{3} c_{5}}}, \\
& l_{1}=\frac{2 \lambda_{1} k_{1}-m_{1}\left(c_{4} \pm \sqrt{c_{4}^{2}-4 c_{3} c_{5}}\right)}{2 c_{3}}, \\
& l_{2}=\frac{-m_{2}\left(c_{4} \pm \sqrt{c_{4}^{2}-4 c_{3} c_{5}}\right)}{2 c_{3}},
\end{aligned}
$$

$$
\begin{aligned}
& \omega_{1}=\frac{\lambda_{1}\left(-\lambda_{1} k_{1} \pm m_{1} \sqrt{c_{4}^{2}-4 c_{3} c_{5}}\right)}{c_{2} c_{3}}, \\
& \omega_{2}=\frac{ \pm \lambda_{1} m_{2} \sqrt{c_{4}^{2}-4 c_{3} c_{5}}}{c_{2} c_{3}}, \\
& k_{2}=\lambda_{3}=\lambda_{4}=\lambda_{6}=\lambda_{7}=0,
\end{aligned}
$$

where $\varepsilon_{1}, \lambda_{1}, k_{1}, m_{1}$, and $m_{2}$ are arbitrary constants and $c_{2} c_{3} \neq 0$ and $c_{4}^{2} \geq 4 c_{3} c_{5}$.

Thus,

$$
u=\frac{k_{1}+\varepsilon_{1} k_{2} \sinh \left(k_{2} x+l_{2} y+m_{2} z+\omega_{2} t\right)}{\left(k_{1} x+l_{1} y+m_{1} z+\omega_{1} t\right)+\varepsilon_{1} \cosh \left(k_{2} x+l_{2} y+m_{2} z+\omega_{2} t\right)},
$$

is a mixed form solution to (5), and $k_{i}, l_{i}, m_{i}$, and $\omega_{i}(i=1,2)$ satisfy (25).

\section{Soliton Solutions}

It is known that many interesting solutions, including breathers, lump, peakon, and rogue solutions, are all special reductions of $\mathrm{N}$-soliton solutions in different situations. This section shows solutions with a few solitons to (5). The standard perturbation method is used to find possible soliton-like solutions. By using a tedious but straightforward calculation, the one-soliton, two-soliton, three-soliton, and $\mathrm{N}$-soliton solutions can be obtained.

3.1. One-Soliton Solution. To obtain the one-soliton solution, we assume that $f$ in (2) is expressed as

$$
f=1+e^{\eta_{1}}
$$

with

$$
\eta_{1}=k_{1} x+p_{1} y+q_{1} z+\omega_{1} t+l_{1},
$$

where $k_{1}, p_{1}, q_{1}, l_{1}$, and $\omega_{1}$ are arbitrary constants. By substituting (27) into (2) and making the coefficients of all exponential terms zero, the dispersion relation is obtained as follows:

$$
\omega_{1}=-\frac{c_{1} k_{1}^{4}+c_{4} p_{1} q_{1}+c_{3} p_{1}^{2}+c_{5} q_{1}^{2}}{c_{2} k_{1}}
$$

Therefore, by substituting (27)-(29) into (4), the onesoliton solution of (5) can be obtained.

3.2. Two-Soliton Solution. To obtain the two-soliton solution, let $f$ in (2) be expressed as 


$$
f=1+e^{\eta_{1}}+e^{\eta_{2}}+\alpha_{12} e^{\eta_{1}+\eta_{2}}
$$

with

$$
\eta_{i}=k_{i} x+p_{i} y+q_{i} z+\omega_{i} t+l_{i}, \quad(i=1,2),
$$

where $k_{i}, p_{i}, q_{i}, l_{i}$, and $\omega_{i}(i=1,2)$ are arbitrary constants. By substituting (30) into (2) and making the coefficients of all exponential terms zero and after some reduction, the followings are obtained:

$$
\begin{aligned}
\omega_{i} & =-\frac{c_{1} k_{i}^{4}+c_{4} p_{i} q_{i}+c_{3} p_{i}^{2}+c_{5} q_{i}^{2}}{c_{2} k_{1}}, \quad(i=1,2), \\
\alpha_{12} & =\frac{3 k_{1}^{2} k_{2}^{2}\left(k_{1}-k_{2}\right)^{2} c_{1}-\left(k_{1} p_{2}-k_{2} p_{1}\right)^{2} c_{3}-\left(k_{1} q_{2}-k_{2} q_{1}\right)\left(k_{1} p_{2}-k_{2} p_{1}\right) c_{4}-\left(k_{1} q_{2}-k_{2} q_{1}\right)^{2} c_{5}}{3 k_{1}^{2} k_{2}^{2}\left(k_{1}+k_{2}\right)^{2} c_{1}-\left(k_{1} p_{2}-k_{2} p_{1}\right)^{2} c_{3}-\left(k_{1} q_{2}-k_{2} q_{1}\right)\left(k_{1} p_{2}-k_{2} p_{1}\right) c_{4}-\left(k_{1} q_{2}-k_{2} q_{1}\right)^{2} c_{5}} .
\end{aligned}
$$

By substituting (30)-(32) into (4), the two-soliton solution of (5) can be obtained.

3.3. Three-Soliton Solution. To obtain the three-soliton solution, it is assumed that $f$ in (2) is expressed as

$$
\begin{aligned}
f= & 1+e^{\eta_{1}}+e^{\eta_{2}}+e^{\eta_{3}}+\alpha_{12} e^{\eta_{1}+\eta_{2}}+\alpha_{13} e^{\eta_{1}+\eta_{3}}+\alpha_{23} e^{\eta_{2}+\eta_{3}} \\
& +\alpha_{123} e^{\eta_{1}+\eta_{2}+\eta_{3}}
\end{aligned}
$$

with

$$
\eta_{i}=k_{i} x+p_{i} y+q_{i} z+\omega_{i} t+l_{i}, \quad(i=1,2,3),
$$

where $k_{i}, p_{i}, q_{i}, l_{i}$, and $\omega_{i}(i=1,2,3)$ are arbitrary constants. Similar to the above method, but through a more complex process and then reduction, the following results are finally obtained:

$$
\begin{aligned}
\omega_{i} & =-\frac{c_{1} k_{i}^{4}+c_{4} p_{i} q_{i}+c_{3} p_{i}^{2}+c_{5} q_{i}^{2}}{c_{2} k_{1}}, \quad(i=1,2,3), \\
\alpha_{i j} & =\frac{3 k_{i}^{2} k_{j}^{2}\left(k_{i}-k_{j}\right)^{2} c_{1}-\left(k_{i} p_{j}-k_{j} p_{i}\right)^{2} c_{3}-\left(k_{i} q_{j}-k_{j} q_{i}\right)\left(k_{i} p_{j}-k_{j} p_{i}\right) c_{4}-\left(k_{i} q_{j}-k_{j} q_{i}\right)^{2} c_{5}}{3 k_{i}^{2} k_{j}^{2}\left(k_{i}+k_{j}\right)^{2} c_{1}-\left(k_{i} p_{j}-k_{j} p_{i}\right)^{2} c_{3}-\left(k_{i} q_{j}-k_{j} q_{i}\right)\left(k_{i} p_{j}-k_{j} p_{i}\right) c_{4}-\left(k_{i} q_{j}-k_{j} q_{i}\right)^{2} c_{5}}, \\
\alpha_{123} & =\alpha_{12} \alpha_{13} \alpha_{23}, \quad(1 \leq i<j \leq 3) .
\end{aligned}
$$

By substituting (33)-(35) into (4), the three-soliton solution of (5) can be obtained. When the three-soliton are substituted into (4) for verification, it is shown that (35) needs to satisfy the constraint condition $c_{4}^{2}=4 c_{3} c_{5}$.

Figure 1 shows the three-soliton solution of (5) through selecting the appropriate parameters. As it is shown in Figure 1, the wave shapes are kink solitons. Both one and two solitons are also kink solitons, whose graphs are omitted here.
3.4. N-Soliton Solution. (5) admits multiple-soliton solution (4) with $f$ of the following form:

$$
f=\sum_{\mu=0,1} \exp \left(\sum_{i=1}^{N} \mu_{i} \eta_{i}+\sum_{1 \leq i<j \leq N}^{N} \mu_{i} \mu_{j} \ln \left(\alpha_{i j}\right)\right),
$$

where

$$
\begin{aligned}
\eta_{i}= & k_{i} x+p_{i} y+q_{i} z+\omega_{i} t+l_{i}, \quad \omega_{i}=-\frac{c_{1} k_{i}^{4}+c_{4} p_{i} q_{i}+c_{3} p_{i}^{2}+c_{5} q_{i}^{2}}{c_{2} k_{1}}, \quad(i=1,2, \ldots, N), \\
\alpha_{i j}= & \frac{3 k_{i}^{2} k_{j}^{2}\left(k_{i}-k_{j}\right)^{2} c_{1}-\left(k_{i} p_{j}-k_{j} p_{i}\right)^{2} c_{3}-\left(k_{i} q_{j}-k_{j} q_{i}\right)\left(k_{i} p_{j}-k_{j} p_{i}\right) c_{4}-\left(k_{i} q_{j}-k_{j} q_{i}\right)^{2} c_{5}}{3 k_{i}^{2} k_{j}^{2}\left(k_{i}+k_{j}\right)^{2} c_{1}-\left(k_{i} p_{j}-k_{j} p_{i}\right)^{2} c_{3}-\left(k_{i} q_{j}-k_{j} q_{i}\right)\left(k_{i} p_{j}-k_{j} p_{i}\right) c_{4}-\left(k_{i} q_{j}-k_{j} q_{i}\right)^{2} c_{5}} \quad(1 \leq i<j \leq N),
\end{aligned}
$$




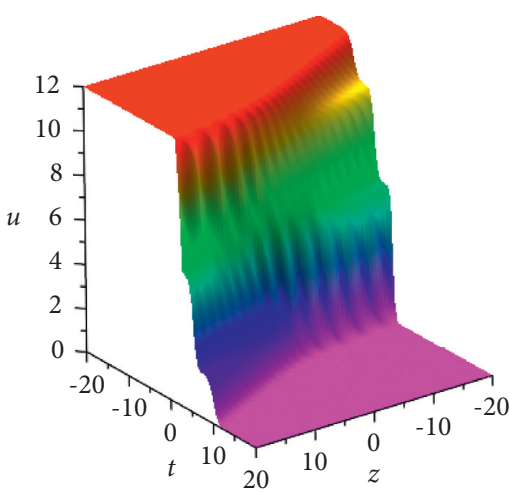

(a)

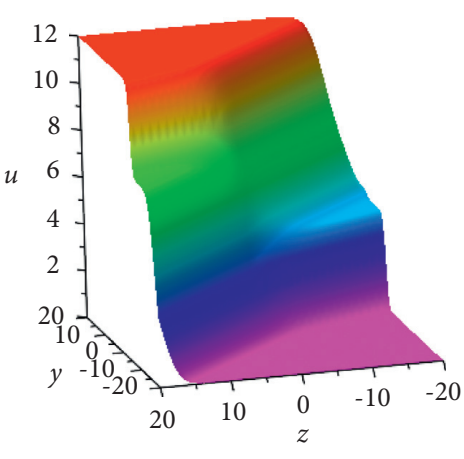

(b)

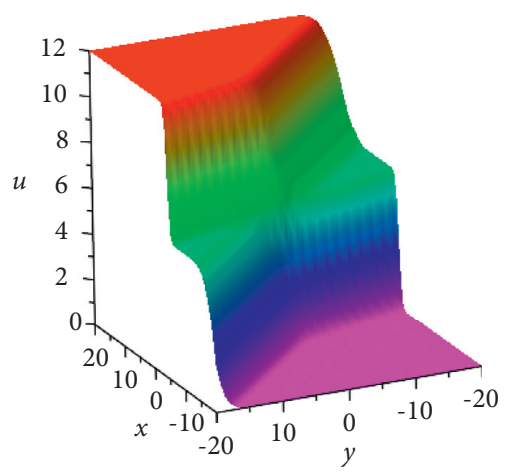

(c)

FIGURE 1: The three-soliton solution for equation (5) by choosing suitable parameters: $k_{1}=2, k_{2}=2, k_{3}=3, p_{1}=1, p_{2}=2$, $p_{3}=1, q_{1}=1, q_{2}=1, q_{3}=1, l_{1}=0, l_{2}=0, l_{3}=0, c_{1}=1, c_{2}=5, c_{3}=1, c_{4}=4$, and $c_{5}=4$. (a) $x=0, y=0$; (b) $x=0, t=0$; (c) $z=0, t=0$.

with $k_{i}, p_{i}, q_{i}$, and $l_{i}$ being arbitrary constants, $c_{4}^{2}=4 c_{3} c_{5}$, and $\sum_{\mu=0,1}$ is the summation that over all possible combinations of $\mu_{i}, \mu_{j}=0,1(i, j=1,2, \ldots, N)$. By substituting (36) and (37) into (4), the $N$-soliton solution of (5) can be obtained.

\section{Lump and Interaction Solutions}

4.1. Lump Solutions. Many studies of the literature have given positive quadratic functions to get lump solutions [15-17]. Based on these studies of the literature, we have the following:

$$
\begin{aligned}
f= & \left(m_{1} x+m_{2} y+m_{3} z+m_{4} t\right)^{2}+\left(n_{1} x+n_{2} y+n_{3} z+n_{4} t\right)^{2} \\
& +f_{0},
\end{aligned}
$$

where $m_{i}, n_{i}(i=1, \ldots, 4)$ and $f_{0}$ are real parameters to be determined.

By substituting $f$ into (2) along with a direct but tedious calculation, 12 sets of solutions are obtained. By removing the redundant parameter solutions, the following three cases are finally obtained.

Case 1

$$
\begin{aligned}
& m_{3}= \pm n_{1}^{2} \sqrt{-\frac{3 c_{1}}{c_{5} f_{0}}}, \\
& m_{4}=\mp \frac{\left(c_{4} n_{2}+2 c_{5} n_{3}\right) m_{3}}{c_{2} n_{1}}, \\
& n_{4}=-\frac{\left(c_{5} n_{3}^{2}+c_{4} n_{3} n_{2}+c_{3} n_{2}^{2}\right) f_{0}+3 c_{1} n_{1}^{4}}{c_{2} n_{1} f_{0}}, \quad m_{1}=m_{2}=0,
\end{aligned}
$$

where $c_{2} n_{1} \neq 0, c_{1} c_{5} f_{0}<0$, and $n_{2}, n_{3}$ are arbitrary constants.

By substituting (38) and (39) into (4), the lump solution of (5) is as follows:

$$
u=\frac{4 n_{1}\left(n_{1} x+n_{2} y+n_{3} z+n_{4} t\right)}{\left(m_{3} z+m_{4} t\right)^{2}+\left(n_{1} x+n_{2} y+n_{3} z+n_{4} t\right)^{2}+f_{0}}
$$

where $m_{i}$ and $n_{i}(i=1, \ldots, 4)$ satisfy (39). 
Case 2

$$
\begin{aligned}
& m_{4}=\mp \frac{m_{3} n_{2} c_{4}+2 n_{2} m_{2} c_{3}+2 n_{3} m_{3} c_{5}+n_{3} m_{2} c_{4}}{c_{2} n_{1}}, \\
& n_{1}=\mp\left(-\frac{\left(c_{5} m_{3}^{2}+c_{4} m_{3} m_{2}+c_{3} m_{2}^{2}\right) f_{0}}{3 c_{1}}\right)^{1 / 4}, \\
& n_{4}= \pm \frac{c_{3}\left(m_{2}^{2}-n_{2}^{2}\right)+c_{4}\left(m_{2} m_{3}-n_{2} n_{3}\right)+c_{5}\left(m_{3}^{2}-n_{3}^{2}\right)}{c_{2} n_{1}}, \quad m_{1}=0
\end{aligned}
$$

where $c_{2} \neq 0, \quad\left(c_{5} m_{3}^{2}+c_{4} m_{3} m_{2}+c_{3} m_{2}^{2}\right) f_{0} c_{1}<0$, and $n_{2}, n_{3}$ are arbitrary constants.

By substituting (38) and (41) into (4), the lump solution of (5) is as follows:

$$
u=\frac{4 n_{1}\left(n_{1} x+n_{2} y+n_{3} z+n_{4} t\right)}{\left(m_{2} y+m_{3} z+m_{4} t\right)^{2}+\left(n_{1} x+n_{2} y+n_{3} z+n_{4} t\right)^{2}+f_{0}},
$$

where $m_{i}$ and $n_{i}(i=1, \ldots, 4)$ satisfy (41). The first set parameter solution in (41) is then chosen. Figure 2 shows the spatial structures of the lump solution and the contour plots for (42) by selecting the appropriate parameter values.

Case 3

$$
\begin{aligned}
& m_{2}=\frac{\Theta_{1} \pm \sqrt{\Theta_{2}}}{2 c_{3} n_{1} f_{0}}, \\
& m_{4}=-\frac{c_{4} n_{3}\left(\Theta_{1} \pm \sqrt{\Theta_{2}}\right) \pm 2 c_{3} n_{2}\left(\Theta_{1} \pm \sqrt{\Theta_{2}}\right)+\Theta_{3}}{2 c_{2} c_{3} n_{1}^{2} f_{0}} \\
& n_{4}=-\frac{3 c_{1}\left(m_{1}^{2}+n_{1}^{2}\right)^{2}+f_{0}\left(c_{5} n_{3}^{2}+c_{4} n_{2} n_{3}+c_{3} n_{2}^{2}\right)}{f_{0} c_{2} n_{1}}
\end{aligned}
$$

where

$$
\begin{aligned}
& \Theta_{1}=\left(c_{4} n_{3} m_{1}-c_{4} n_{1} m_{3}+2 c_{3} n_{2} m_{1}\right) f_{0}, \\
& \Theta_{2}=-12 c_{1} c_{3} c_{5} f_{0}\left(n_{1}^{2}+m_{1}^{2}\right)^{3}-c_{5} f_{0}^{2}\left(n_{3} m_{1}-n_{1} m_{3}\right)^{2}\left(4 c_{3} c_{5}-c_{4}^{2}\right), \\
& \Theta_{3}=-3 c_{1} c_{5} m_{1}\left(n_{1}^{2}+m_{1}^{2}\right)^{2}+c_{4} c_{5}^{2} n_{2}\left(n_{1} m_{3}-n_{3} m_{1}\right) f_{0}+c_{5}^{2}\left(2 c_{5} m_{3} n_{3} n_{1}-c_{5} n_{3}^{2} m_{1}-n_{2}^{2} m_{1} c_{3}\right) f_{0},
\end{aligned}
$$

where $c_{2} c_{3} n_{1} f_{0} \neq 0, \Theta_{2} \geq 0$, and $m_{1}, m_{3}, n_{2}$, and $n_{3}$ are arbitrary constants.

By substituting (38) and (43) into (4), the lump solution of (5) is obtained as follows:

$u=\frac{4 m_{1}\left(m_{1} x+m_{2} y+m_{3} z+m_{4} t\right)+4 n_{1}\left(n_{1} x+n_{2} y+n_{3} z+n_{4} t\right)}{\left(m_{1} x+m_{2} y+m_{3} z+m_{4} t\right)^{2}+\left(n_{1} x+n_{2} y+n_{3} z+n_{4} t\right)^{2}+f_{0}}$,

where $m_{i}$ and $n_{i}(i=1, \ldots, 4)$ satisfy (43).

It is noteworthy to state that the spatial structures of the lump solutions in Case 1 and Case 3 are like those in Case 2. From the above three cases, the obtained lump waves have the following asymptotic behavior: $\lim _{x \longrightarrow \pm \infty} u=0$, $\lim _{t \longrightarrow \infty} u=0, \lim _{y \longrightarrow \pm \infty} u=0$, and $\lim _{z \longrightarrow \pm \infty} u=0$.

4.2. Interaction Solution between Solitary and Lump Waves. To construct interaction solutions, the following form is taken:

$$
f=a \xi_{1}^{2}+b \xi_{2}^{2}+k g\left(\xi_{3}\right)+c
$$

with

$$
\begin{aligned}
& \xi_{1}=m_{1} x+m_{2} y+m_{3} z+m_{4} t, \\
& \xi_{2}=n_{1} x+n_{2} y+n_{3} z+n_{4} t, \\
& \xi_{3}=k_{1} x+k_{2} y+k_{3} z+k_{4} t,
\end{aligned}
$$

where $a, b, k, c, m_{i}, n_{i}$, and $k_{i}(i=1, \ldots, 4)$ are arbitrary parameters and $g$ is an arbitrary function. By choosing different functions for $g$, abundant interaction solutions can be obtained [18-21].

This section mainly considers the interaction between the lump and the soliton solutions and then discusses their corresponding dynamical behaviors. The following values are chosen for $a=1$ and $b=c=0$ in (46) to obtain

$$
\begin{aligned}
f= & \left(m_{1} x+m_{2} y+m_{3} z+m_{4} t\right)^{2} \\
& +k \cosh \left(k_{1} x+k_{2} y+k_{3} z+k_{4} t\right) .
\end{aligned}
$$

By substituting (48) into (2) and combining direct but tedious calculations, 31 sets of solutions are obtained for the above undetermined parameters. To ensure the generality of parametric solutions, the following four cases are obtained.

Case 4

$$
\begin{aligned}
& k_{4}=-\frac{k_{2}^{2} c_{3}+c_{4} k_{2} k_{3}+c_{5} k_{3}^{2}}{c_{2} k_{1}}, \\
& m_{4}=-\frac{\left(c_{4} k_{2}+2 c_{5} k_{3} \pm k_{2} \sqrt{c_{4}^{2}-4 c_{3} c_{5}}\right)^{2} m_{1}}{4 c_{2} c_{5} k_{1}^{2}}, \\
& m_{3}= \pm \frac{\left(c_{4} k_{2}+2 c_{5} k_{3} \pm k_{2} \sqrt{c_{4}^{2}-4 c_{3} c_{5}}\right) m_{1}}{2 c_{5} k_{1}}, \quad c_{1}=m_{2}=0,
\end{aligned}
$$

where $c_{2} c_{5} k_{1} \neq 0, c_{4}^{2} \geq 4 c_{3} c_{5}$, and $k, k_{2}, k_{3}$, and $m_{1}$ are arbitrary constants. 


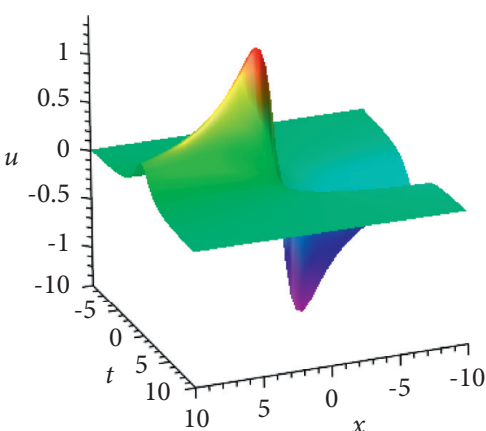

(a)

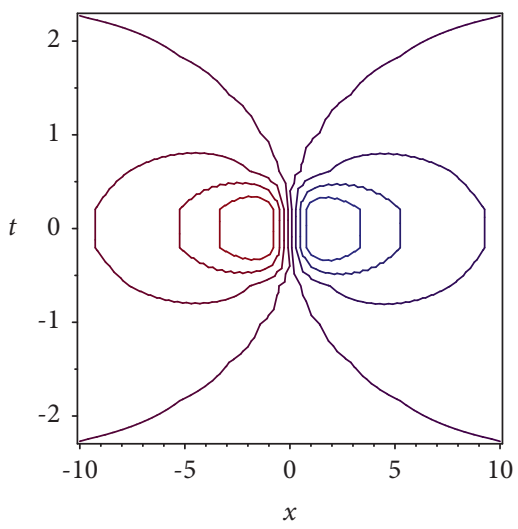

(d)

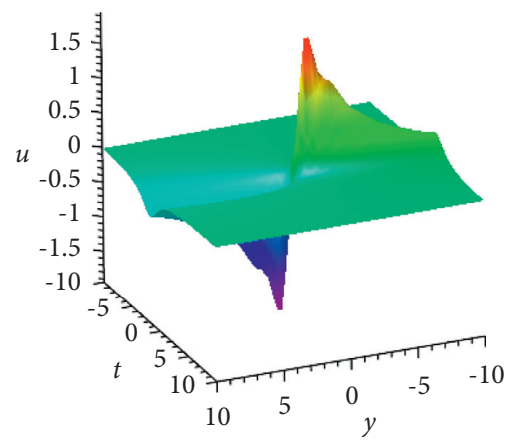

(b)

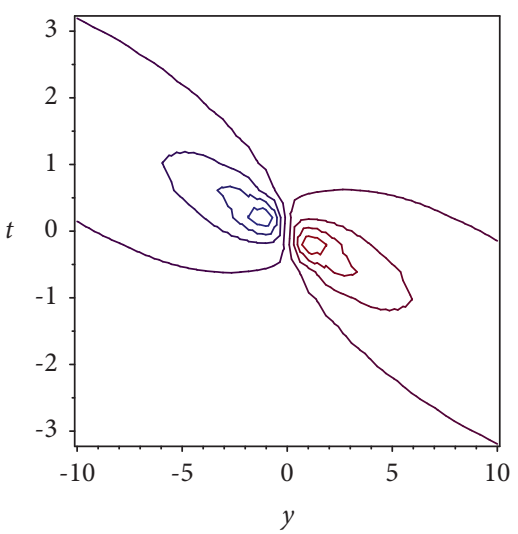

(e)

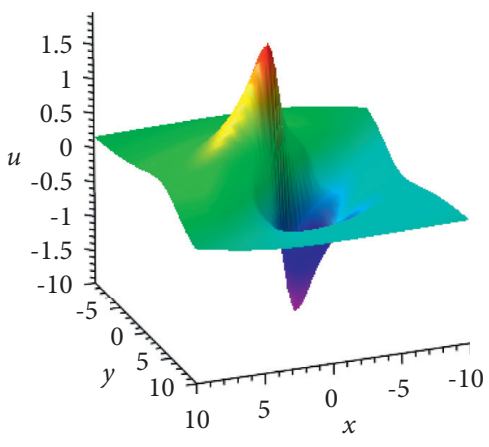

(c)

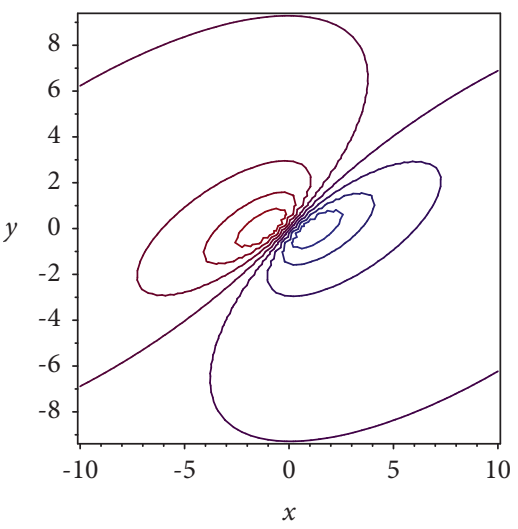

(f)

Figure 2: Spatial structures of lump solution (42) with parameters: $c_{1}=-1$ and $c_{2}=c_{3}=c_{4}=c_{5}=m_{2}=m_{3}=n_{2}=n_{3}=f_{0}=1$. (a) $y=0, z=0$; (b) $x=0, z=0$; (c) $z=0, t=0$. (d-f) Contour plots corresponding to subfigures (a-c), respectively.

By substituting (48) and (49) into (4), the interaction solution of (5) is obtained as follows:

$$
u=\frac{4 m_{1}\left(m_{1} x+m_{3} z+m_{4} t\right)+2 k k_{1} \sinh \left(k_{1} x+k_{2} y+k_{3} z+k_{4} t\right)}{\left(m_{1} x+m_{3} z+m_{4} t\right)^{2}+k \cosh \left(k_{1} x+k_{2} y+k_{3} z+k_{4} t\right)}
$$

where $m_{i}$ and $k_{i}(i=1, \ldots, 4)$ satisfy (49) and the first set parameter solution in (49) is chosen. Figure 3 shows the spatial structures of the interaction solution and the contour plots for (50) by selecting the appropriate parameter values. Interestingly, it was found that Figure 3(a) is consistent with the result of the interaction between the kink soliton and the lump waves (see Figures 1 and 2).

\section{Case 5}

$$
\begin{aligned}
& k_{1}=\frac{k_{2} m_{1}}{m_{2}}, \\
& k_{3}=\frac{k_{2} m_{3}}{m_{2}}, \\
& k_{4}=-\frac{k_{2}\left(c_{3} m_{2}^{2}+c_{4} m_{3} m_{2}+c_{5} m_{3}^{2}\right)}{c_{2} m_{1} m_{2}}, \\
& m_{4}=-\frac{c_{3} m_{2}^{2}+c_{4} m_{3} m_{2}+c_{5} m_{3}^{2}}{c_{2} m_{1}}, \quad c_{1}=0,
\end{aligned}
$$

where $c_{2} m_{1} m_{2} \neq 0$, and $k, k_{2}, m_{3}$, and $c_{i}(i=3,4,5)$ are arbitrary constants.

By substituting (48) and (51) into (4), the interaction solution of (5) is as follows:

$$
u=\frac{4 m_{1}\left(m_{1} x+m_{2} y+m_{3} z+m_{4} t\right)+2 k k_{1} \sinh \left(k_{1} x+k_{2} y+k_{3} z+k_{4} t\right)}{\left(m_{1} x+m_{2} y+m_{3} z+m_{4} t\right)^{2}+k \cosh \left(k_{1} x+k_{2} y+k_{3} z+k_{4} t\right)},
$$

where $m_{i}$ and $k_{i}(i=1, \ldots, 4)$ satisfy (51). Figure 4 shows the spatial structures of the interaction solution and the contour plots for (52) by selecting the appropriate parameter values.

Case 6

$$
\begin{aligned}
& k_{4}=-\frac{c_{3} k_{2}^{2}+c_{4} k_{3} k_{2}+c_{5} k_{3}^{2}}{c_{2} k_{1}}, \\
& m_{3}=\frac{m_{2}\left(-c_{4} \pm \sqrt{c_{4}^{2}-4 c_{3} c_{5}}\right)}{2 c_{5}}, \\
& m_{4}=\frac{m_{2} \sqrt{c_{4}^{2}-4 c_{3} c_{5}}\left(k_{2} \sqrt{c_{4}^{2}-4 c_{3} c_{5}} \mp\left(c_{4} k_{2}+2 c_{5} k_{3}\right)\right)}{2 c_{2} c_{5} k_{1}}, \\
& c_{1}=m_{1}=0,
\end{aligned}
$$




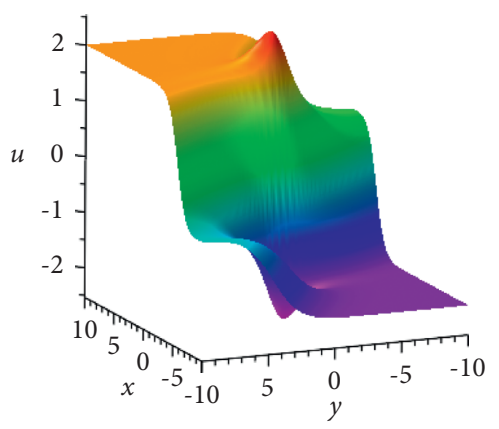

(a)

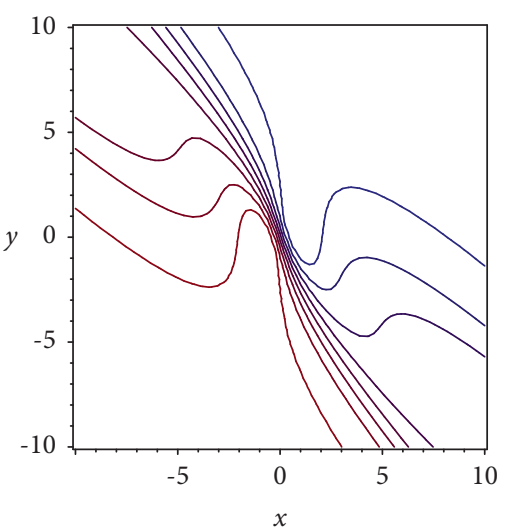

(d)

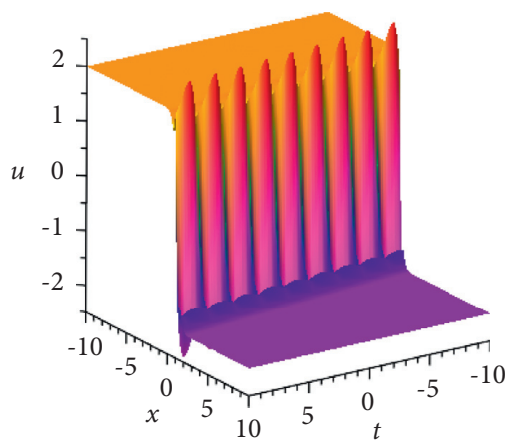

(b)

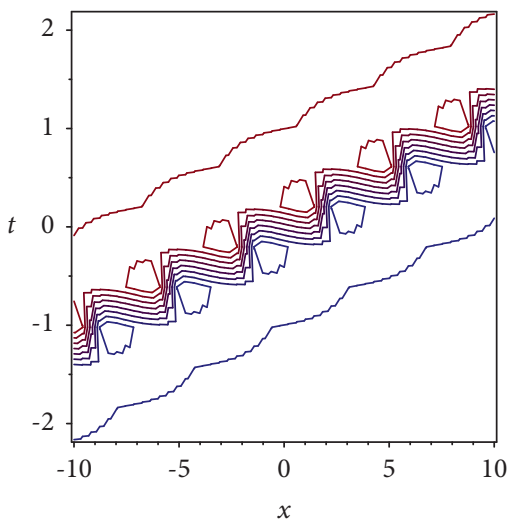

(e)

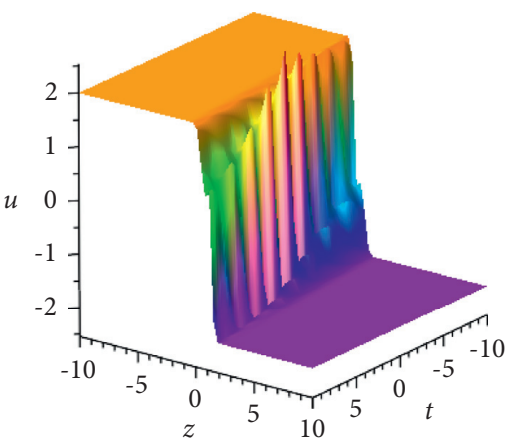

(c)

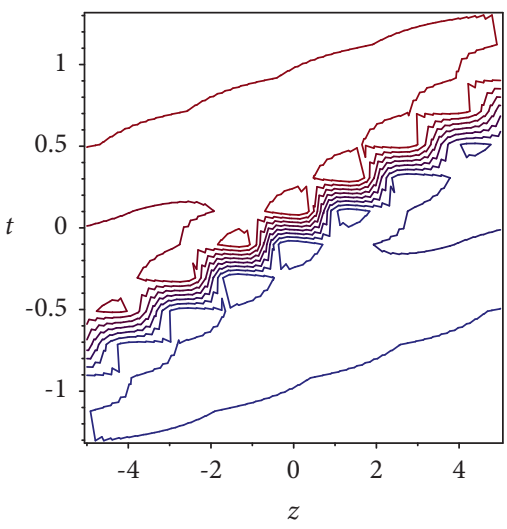

(f)

FIGURE 3: Spatial structures of interaction solution (50) with parameters: $c_{2}=1, c_{3}=1, c_{4}=4, c_{5}=4$, and $m_{1}=k_{1}=k_{2}=k_{3}=k_{4}=1$. (a) $z=0, t=0$; (b) $y=0, z=0$; (c) $x=0, y=0$. (d-f) Contour plots corresponding to subfigures (a-c), respectively.

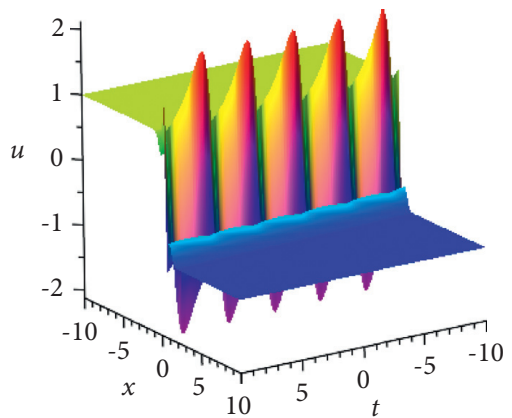

(a)

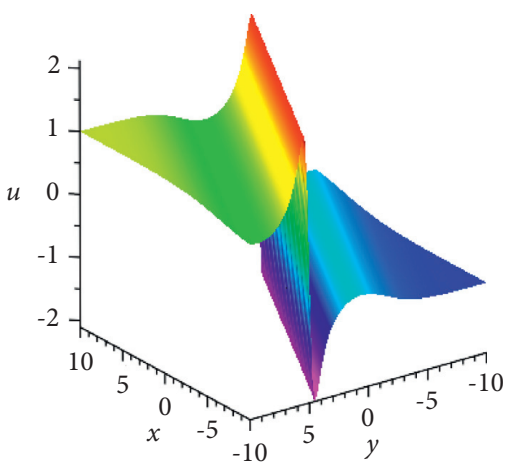

(c)

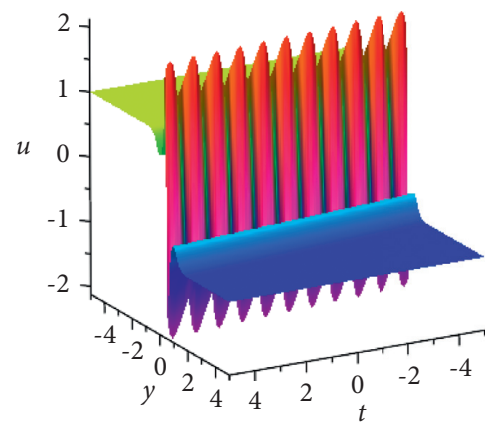

(b)

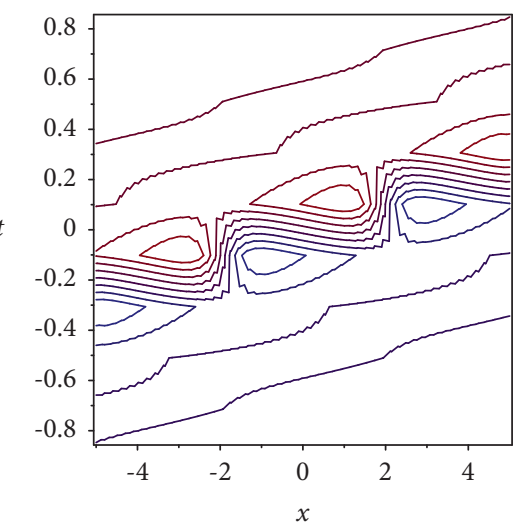

(d)

FIGURE 4: Continued. 


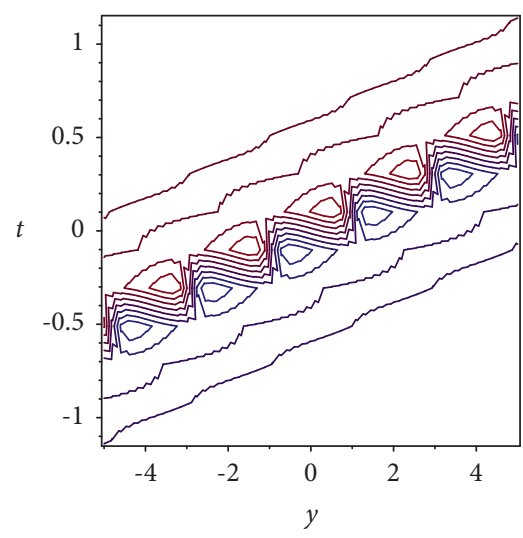

(e)

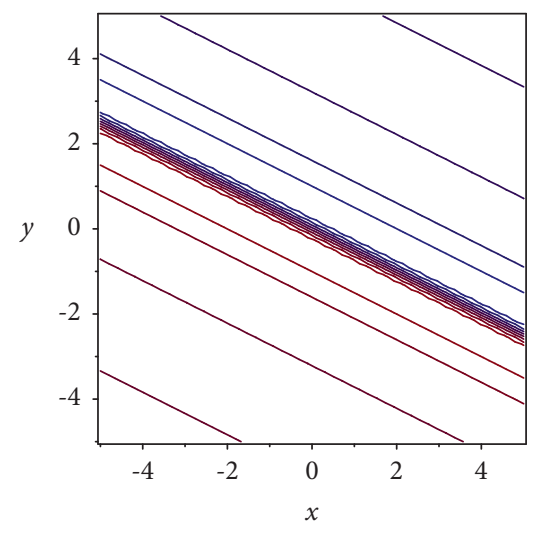

(f)

Figure 4: Spatial structures of interaction solution (52) with parameters: $c_{2}=c_{3}=c_{4}=c_{5}=k_{1}=k_{2}=1, m_{1}=1, m_{2}=2$, and $m_{3}=3$. (a) $y=0, z=0$; (b) $x=0, z=0$; (c) $z=0, t=0$. (d-f) Contour plots corresponding to subfigures (a-c), respectively.

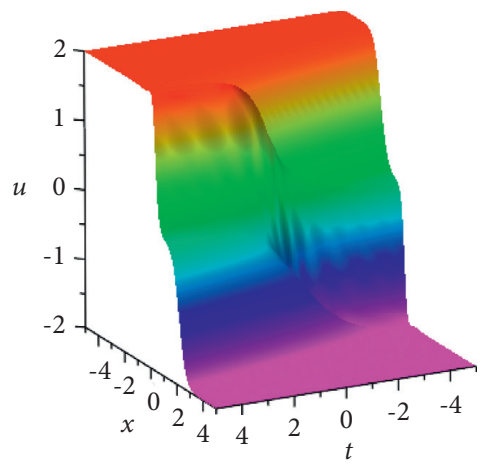

(a)

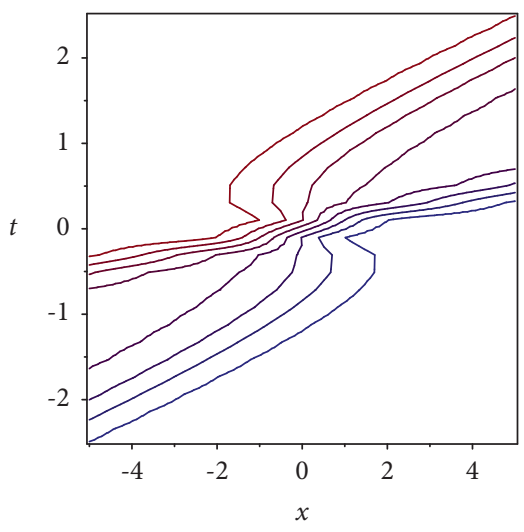

(d)

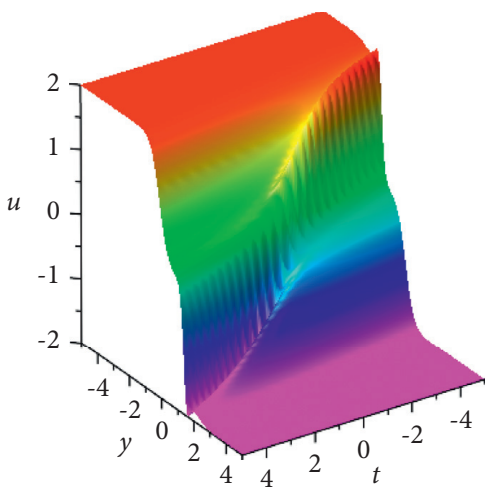

(b)

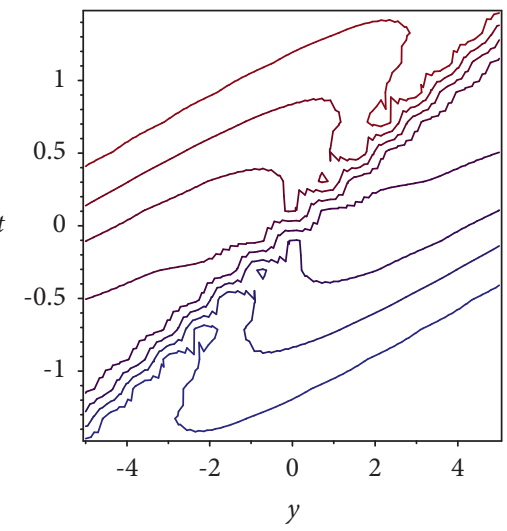

(e)

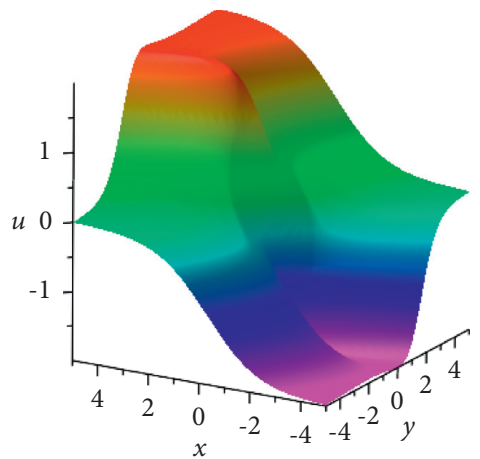

(c)

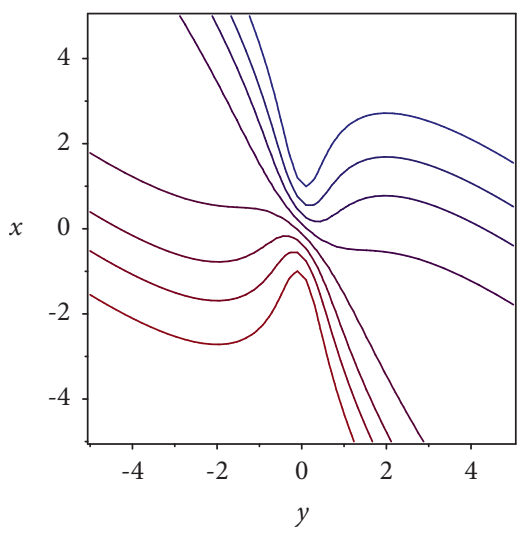

(f)

FIGURE 5: Spatial structures of interaction solution (54) with parameters: $c_{2}=c_{3}=c_{5}=k_{1}=k_{2}=k_{3}=k=1, c_{4}=3$, and $m_{2}=2$. (a) $y=0, z=0$; (b) $x=0, z=0$; (c) $z=0, t=0$. (d-f) Contour plots corresponding to subfigures (a-c), respectively.

where $c_{2} c_{5} k_{1} \neq 0, c_{4}^{2} \geq 4 c_{3} c_{5}$, and $k, k_{2}$, and $k_{3}$ are arbitrary constants.

By substituting (48) and (53) into (4), the interaction solution of (5) is obtained as follows:

$$
u=\frac{2 k k_{1} \sinh \left(k_{1} x+k_{2} y+k_{3} z+k_{4} t\right)}{\left(m_{2} y+m_{3} z+m_{4} t\right)^{2}+k \cosh \left(k_{1} x+k_{2} y+k_{3} z+k_{4} t\right)},
$$




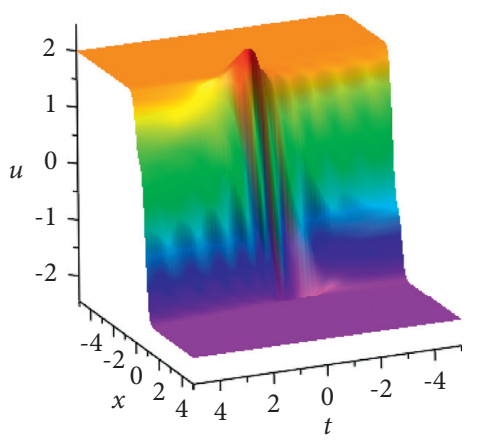

(a)

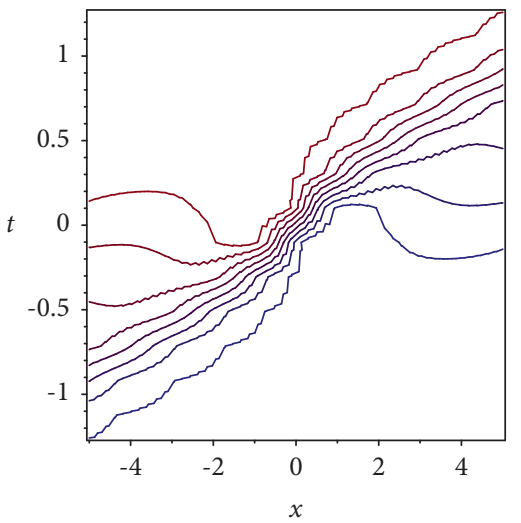

(d)

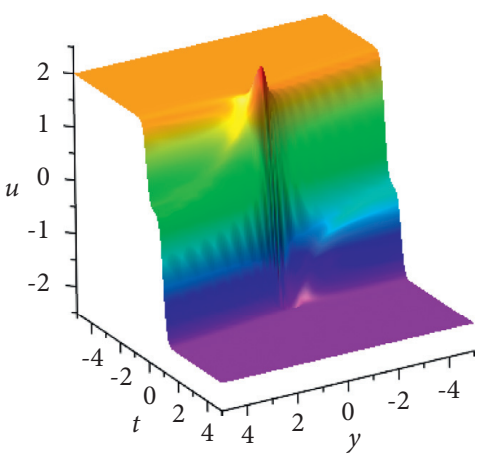

(b)

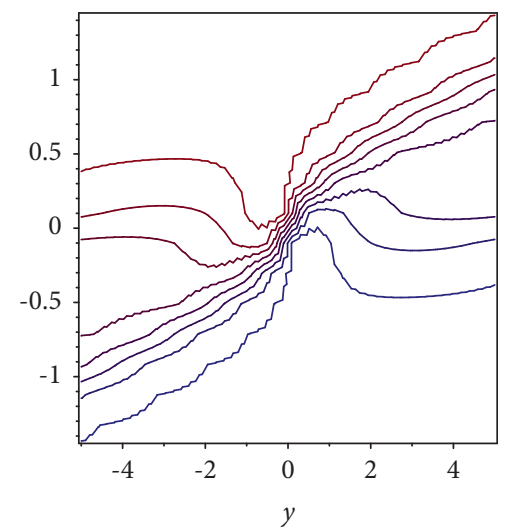

(e)

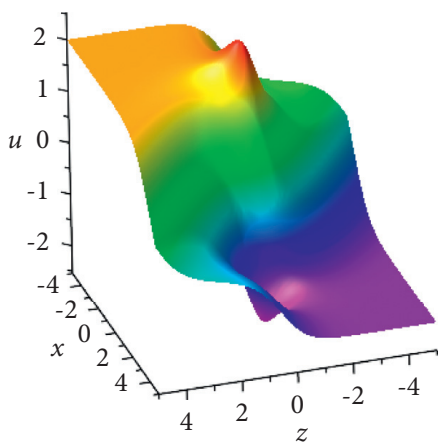

(c)

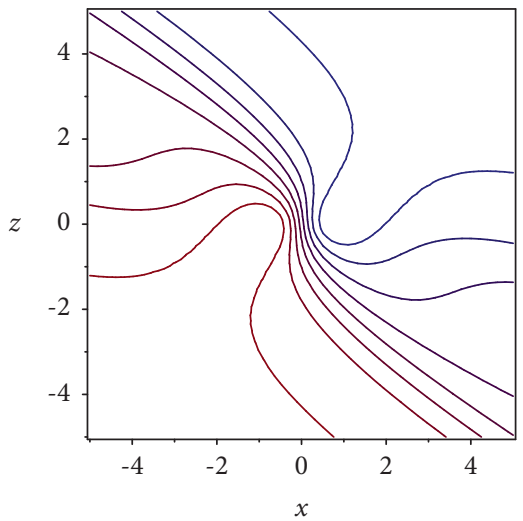

(f)

FIGURE 6: Spatial structures of interaction solution (56) with parameters: $c_{2}=c_{3}=c_{5}=k_{1}=k_{2}=k_{3}=k=m_{1}=1, c_{4}=3$, and $m_{2}=2$. (a) $y=0, z=0$; (b) $x=0, z=0$; (c) $y=0, t=0$. (d-f) Contour plots corresponding to subfigures (a-c), respectively.

where $m_{i}, k_{i}(i=1, \ldots, 4)$ satisfy $(53)$. The first set parameter solution in (53) is then chosen. Figure 5 shows the spatial structures of the interaction solution and the contour plots for (54) by selecting the appropriate parameter values.

\section{Case 7}

$$
\begin{aligned}
& k_{3}=\frac{-c_{4} k_{2} m_{1} \pm \sqrt{c_{4}^{2}-4 c_{3} c_{5}}\left(2 k_{1} m_{2}-k_{2} m_{1}\right)}{2 c_{5} m_{1}}, \\
& k_{4}=\frac{m_{2}\left(-c_{4}^{2}+4 c_{3} c_{5}\right)\left(k_{1} m_{2}-k_{2} m_{1}\right)}{c_{2} c_{5} m_{1}^{2}}, \\
& m_{3}=\frac{m_{2}\left(-c_{4} \pm \sqrt{c_{4}^{2}-4 c_{3} c_{5}}\right)}{2 c_{5}}, \quad c_{1}=m_{4}=0
\end{aligned}
$$

where $c_{2} c_{5} m_{1} \neq 0, c_{4}^{2} \geq 4 c_{3} c_{5}$, and $k, k_{2}, m_{1}$, and $m_{2}$ are arbitrary constants.

By substituting (48) and (55) into (4), the interaction solution of (5) is obtained as follows:

$u=\frac{4 m_{1}\left(m_{1} x+m_{2} y+m_{3} z\right)+2 k k_{1} \sinh \left(k_{1} x+k_{2} y+k_{3} z+k_{4} t\right)}{\left(m_{1} x+m_{2} y+m_{3} z\right)^{2}+k \cosh \left(k_{1} x+k_{2} y+k_{3} z+k_{4} t\right)}$, where $m_{i}, k_{i}(i=1, \ldots, 4)$ satisfies (55). The first set parameter solution in (55) is then chosen. Figure 6 shows the spatial structures of the interaction solution and the contour plots for (56) by selecting the appropriate parameter values.

\section{Conclusion}

In this article, a generalized $(3+1)$-dimensional NLEE whose bilinear equation is obtained by a multivariable polynomial was discussed. Based on the bilinear equation, the BBT is given and it consists of four bilinear equations involving seven arbitrary parameters except for the parameters of the equation itself. By using the BBT, two traveling wave solutions and a mixed solution were obtained. Also, the $\mathrm{N}$-soliton solutions were constructed and the dynamic properties of the soliton solutions were shown graphically. Furthermore, the lump and the interaction solutions were constructed. The properties of the explicit analytic solutions were shown by using figures. The general expression of the interaction solution was also given. By determining the different forms of the arbitrary function, more abundant interaction solutions will be obtained. Hence, the interaction between the lump and other solutions, including periodic solution and kink solution, can further be explored. 
Finally, this article not only presents a new example for $(3+1)$-dimensional NLEE but also provides a reference for finding the lump and the interaction solutions of other generalized multidimensional NLEEs.

\section{Data Availability}

The Maple data used to support the findings of this study are available from the corresponding author upon request.

\section{Conflicts of Interest}

The authors declare that they have no conflicts of interest.

\section{Acknowledgments}

This work was completed with the support of the National Natural Science Foundation of China (12001480 and 12071418), the Scientific and Technological Innovation Programs of Higher Education Institutions in Shanxi (2019L0868), the Applied Basic Research Program of Shanxi Province (20210302123082 and 201901D211461), and the Doctoral Research Project of Yuncheng University (YQ2020019). The authors would like to express their gratitude to EditSprings (https://www.editsprings.com/) for the expert linguistic services provided.

\section{References}

[1] M. J. Ablowitz and P. A. Clarkson, Soliton, Nonlinear Evolution Equations and Inverse Scttering, Cambridge University Press, Cambridge, UK, 1991.

[2] R. Hirota, Direct Method in Soliton Theory, Cambridge University Press, Cambridge, UK, 2004.

[3] V. B. Matveev and M. A. Salle, Darboux Transformation and Solitons, Springer-Verlag, Berlin, Germany, 1991.

[4] S. L. Zhang, S. Y. Lou, and C. Z. Qu, "Variable separation and exact solutions to generalized nonlinear diffusion equations," Chinese Physics Letters, vol. 12, p. 1741, 2002.

[5] J.-H. He and X.-H. Wu, "Exp-function method for nonlinear wave equations," Chaos, Solitons \& Fractals, vol. 30, no. 3, pp. 700-708, 2006.

[6] X. B. Hu and H. W. Tam, "Application of Hirota's bilinear formalism to a two-dimensional lattice by Leznov," Physics Letters A, vol. 276, no. s1-4, pp. 65-72, 2000.

[7] R. X. Yao, X. Y. Jiao, and S. Y. Lou, "A maple package to compute lie symmetry groups and symmetry reductions of $(1+1)$-dimensional nonlinear systems," Computational and Theoretical Physics, vol. 25, no. 6, pp. 1927-1930, 2008.

[8] S. Y. Lou, X. Hu, and Y. Chen, "Nonlocal symmetries related to Bäcklund transformation and their applications," Journal of Physics A: Mathematical and Theoretical, vol. 45, no. 15, Article ID 155209, 2012.

[9] T. Xu, H. Li, H. Zhang, and M. Li, S. Lan, Darboux transformation and analytic solutions of the discrete PT-symmetric nonlocal nonlinear Schrödinger equation," Applied Mathematics Letters, vol. 63, pp. 88-94, 2017.

[10] W. X. Ma, Y. Zhang, Y. N. Tang, and J. Y. Tu, "Hirota bilinear equations with linear subspaces of solutions," Applied Mathematics and Computation, vol. 218, pp. 7174-7183, 2012.

[11] B. Tian and Y. T. Gao, "Variable-coefficient balancing-act method and variable-coefficient $\mathrm{KdV}$ equation from fluid dynamics and plasma physics," European Physical Journal B, vol. 22, no. 3, pp. 351-360, 2001.

[12] L. Xing, B. Tian, H. Q. Zhang, X. Tao, and H. Li, "Generalized $(2+1)$-dimensional Gardner model: bilinear equations, Bäcklund transformation, Lax representation and interaction mechanisms," Nonlinear Dynamics, vol. 67, no. 3, pp. 2279-2290, 2012.

[13] R. Guo, Y. F. Liu, H. Q. Hao, and F.-H. Qi, "Coherently coupled solitons, breathers and rogue waves for polarized optical waves in an isotropic medium," Nonlinear Dynamics, vol. 80, no. 3, pp. 1221-1230, 2015.

[14] J. M. Tu, S. F. Tian, M. J. Xu, and X.-Q. Song, "Bäcklund transformation, infinite conservation laws and periodic wave solutions of a generalized $(3+1)$-dimensional nonlinear wave in liquid with gas bubbles," Nonlinear Dynamics, vol. 83, no. 3, pp. 1199-1215, 2016.

[15] P. A. Clarkson and E. Dowie, "Rational solutionas of the Boussinesq equation and applications to rogue waves," Transactions of Mathematics and Its Applications, vol. 1, no. 1, Article ID tnx003, 2017.

[16] P. Gaillard, "Families of quasi-rational solutions of the NLS equation and multi-rogue waves," Journal of Physics A, vol. 44, Article ID 435204, 2011.

[17] Y. Yue, L. Huang, and Y. Chen, "N-solitons, breathers, lumps and rogue wave solutions to a $(3+1)$-dimensional nonlinear evolution equation," Computers and Mathematics Applications, vol. 75, pp. 2538-2548, 2018.

[18] Z. Lu, E. M. Tian, and R. Grimshaw, "Interaction of two lump solitons described by the Kadomtsev-Petviashvili I equation," Wave Motion, vol. 40, pp. 123-135, 2004.

[19] X. W. Yan, S. F. Tian, M. J. Dong, and L. zou, "Bäcklund transformation, rogue wave solutions and interaction phenomena for a $(3+1)$-dimensional B-type Kadomtsev-Petviashvili-Boussinesq equation," Nonlinear Dynamics, vol. 92, pp. 709-720, 2018.

[20] A. S. Fokas, D. E. Pelinovsky, and C. Sulem, "Interaction of lumps with a line soliton for the DSII equation," Physica D, vol. 152-153, pp. 189-198, 2001.

[21] Y. N. Tang, S. Q. Tao, and Q. Guan, "Lump solitons and the interaction phenomena of them for two classes of nonlinear evolution equations," Computers and Mathematics Applications, vol. 72, no. 9, pp. 2334-2342, 2016. 